\title{
RECENT OPERATIONAL DATA ON CONTINUOUS TOP-UP OPERATION AT THE ADVANCED PHOTON SOURCE*
}

\author{
L. Emery ${ }^{\dagger}$, Advanced Photon Source, Argonne National Laboratory, Argonne, IL
}

\begin{abstract}
Since June 2000, there have been three 6-day or 13-day periods of continuous top-up operations at the Advanced Photon Source, totaling four weeks. We will report on injection and storage ring parameters and present a statistical analysis of $\mathrm{x}$-ray beam stability.
\end{abstract}

\section{INTRODUCTION}

The Advanced Photon Source has been providing beam for X-ray users in top-up mode for several 6-day or 13-day periods since June 2000. Our last top-up running period was in March 2001, and our next one starts in mid-June 2001. Our configuration and procedures for top-up operation have been essentially the same since the beginning.

\section{RUNNING PARAMETERS}

We maintain a total current of $102 \pm 0.2 \mathrm{~mA}$ by injecting a single bunch from the $7-\mathrm{GeV}$ injector into the ring every two minutes. The two-minute interval is strictly enforced to allow the users a consistent timeframe for an upcoming injection. If a pulse from the injector fails, then we must wait two minutes for the next injection.

Automatic processes control the timing of the injection and select the bucket in which to inject the single bunch. The bucket number at every injection pulse is determined from a continual measurement of the bunch charges and a comparison with the desired bunch pattern, which can be made arbitrarily, as one wishes. The bunch pattern we have been using in the past year was a 12-mA bunch train of 6 consecutive buckets followed by 21 single bunches separated by $150 \mathrm{~ns}$ for the other $88 \mathrm{~mA}$. Presently, we fill 100 $\mathrm{mA}$ in 23 bunches spaced by $150 \mathrm{~ns}$, and we will use this bunch pattern for the top-up run in June 2001.

The control system gives a warning several seconds before injection for the convenience of users, and also to allow background processes to initiate the startup of the electron source of the injectors in preparation for injection.

For a 20-h stored beam lifetime (our beam emittances are $7.5 \mathrm{~nm}$-rad and $75 \mathrm{pm}$-rad in the horizontal and vertical planes, respectively), the injectors are required to deliver a charge of more than $0.6 \mathrm{nC}$ every two minutes to maintain the current in the ring. We normally set the injectors to deliver $1 \mathrm{nC}$ to insure against an occasional lost pulse. At the

\footnotetext{
* Work supported by U.S. Department of Energy, Office of Basic Energy Sciences under Contract No. W-31-109-ENG-38.

† emery@aps.anl.gov
}

end of each two-minute interval, injection is enabled only if the stored beam drops below the target current of $102 \mathrm{~mA}$. Thus, the current reading can fluctuate around the target current more or less depending on the amount of charge prepared for injection. Fig. 1 shows a histogram of the stored current for the three top-up runs.

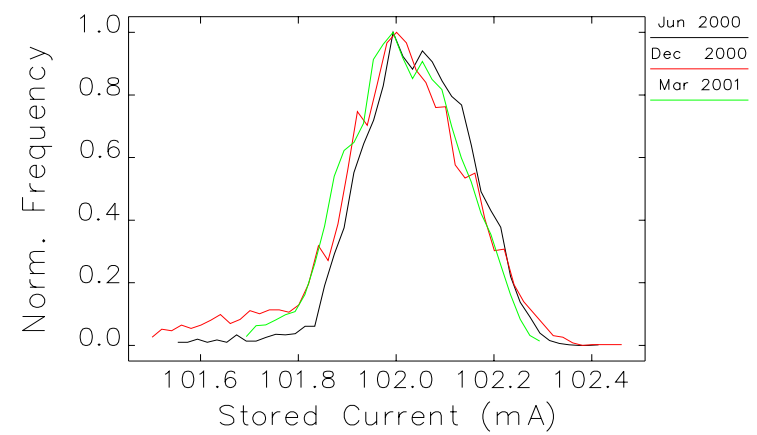

Figure 1: Histogram of stored current for the first two topup runs with outliers from injector down-times excluded.

The two-minute interval was approved by consensus by the users. It also fits well within the constraints of our injector and stored beam lifetime. If we operate in the future with a lattice or fill pattern with much lower lifetime, injector capacity will have to be increased. The possibility of injecting a rapid sequence of weaker pulses every two minutes was not considered desirable by the users.

We inject with mismatch kickers, which splits the injection offset between the injection beam and the stored beam, thus improving capture efficiency to the point of almost no losses at the narrow-gap insertion device chambers. The mismatch produces an average beam motion of $2.6 \mathrm{~mm}$, which decoheres into a factor of 36 emittance blowup. Users have the ability to exclude this period from their data by a gate provided by the control system. To control injection launching errors, a workstation-based feedback runs on the transfer line to maintain the trajectory. For the infrequent injections of top-up mode, this feedback requires a few seconds of injector beam in advance for some averaging to occur and to make a correction to the trajectory before a pulse is injected into the ring.

Capture efficiency is especially relevant in top-up operation because open photon shutters allow the bremsstrahlung from lost injection particles to be transported into the frontend enclosures of the experimental floor. Incidentally, when filling the ring from, say $60 \mathrm{~mA}$ to $100 \mathrm{~mA}$, in the standard refill operation mode the shutters are left open to maintain as much thermal stability as possible. Safety- 
system radiation monitors positioned outside each beamline front-end enclosures detect little activity when injection is optimized and shutters are open. Using the radiation monitor readbacks during refill operations at the worst location, the daily dose is estimated to be about $0.1 \mathrm{mrem}$. We expect about the same daily dose during top-up operation, as long as injection losses are kept low.

We estimated about $1 \%$ loss when the transfer line is optimized. The residual beam loss after optimization can probably be reduced if the relatively large booster beam emittance (130 nm-rad) was somehow reduced.

A Cerenkov detector installed at all insertion device vacuum chambers gives a sensitive real-time measurement of the losses at each injection pulse. The readback can be monitored for unusual losses. The losses (as a function of passes around the ring) give clues on how to make an adjustment to recover the optimum injection conditions. (The time structure for each misteering has to be empirically determined in advance.)

\section{PERFORMANCE EVALUATION}

It is expected that the constant level of heat and electronic signals in top-up mode improves photon beam position stability on the time scale of minutes to days. We have some anecdotal reports from users of improved stability. However, we have no systematic way to demonstrate the stability of $\mathrm{x}$-ray beams outside of the tunnel enclosure. All users have a different experimental setup and have different sensitivities to position fluctuations.

For now we examine and compare the accelerator data for the refill and top-up modes and determine whether stability is improved in top-up mode.

For characterizing slow beam drift, the available relevant data are the dipole beamline $\mathrm{x}$-ray beam position monitors (XBPM) and the corrector dipoles in orbit correction. There are two XBPM for each dipole source beamline located $11 \mathrm{~m}$ and $18 \mathrm{~m}$ downstream, and they read only the vertical position. The beam drift in the horizontal plane is characterized by the horizontal correctors. We do not use the insertion device XBPMs because they are highly gapdependent.

Orbit correction is configured with almost all of the storage ring rf BPMs and two correctors per plane. The process is workstation-based and runs continuously with an interval of $4 \mathrm{~s}$ (sometimes $2.5 \mathrm{~s}$ ). The correctors will respond not only to real magnetic field changes (e.g., from an insertion device), but also to any BPM electronic offset variation and any BPM displacement, which are unpredictable and not negligible. The results of these errors are seen as slow variations of XBPM or corrector readbacks over a period of a week, either in refill or top-up mode as shown in Figs. 2 and 3. Top-up mode is expected to reduce the drift seen in these types of plots, assuming that they are caused by intensity-related effects.

Because of the large quantities of data, a statistical analysis is done to compare the two modes of operation. Special
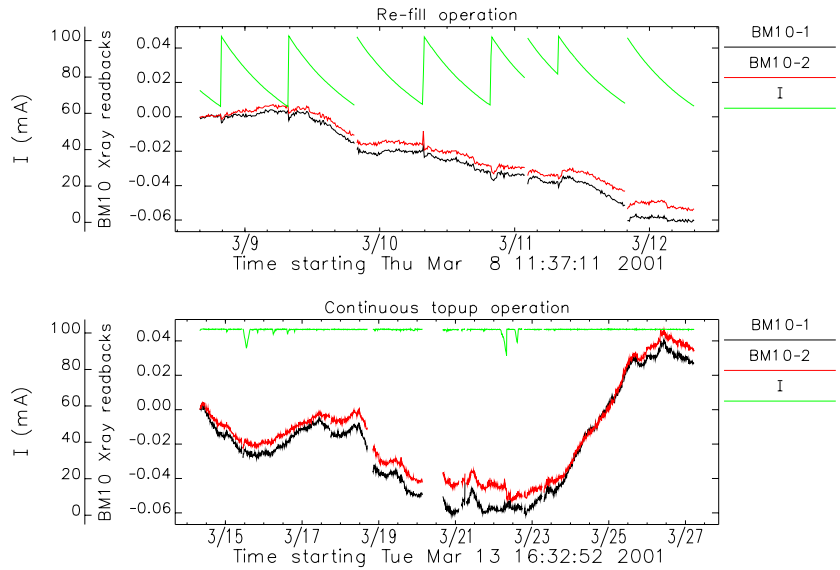

Figure 2: Example of slow variations of XBPMs (sector 10) during a 6-day refill period and the most recent top-up period of March 2001. The worsening of the drift during top-up operation is unexpected, and is referred to in the text.

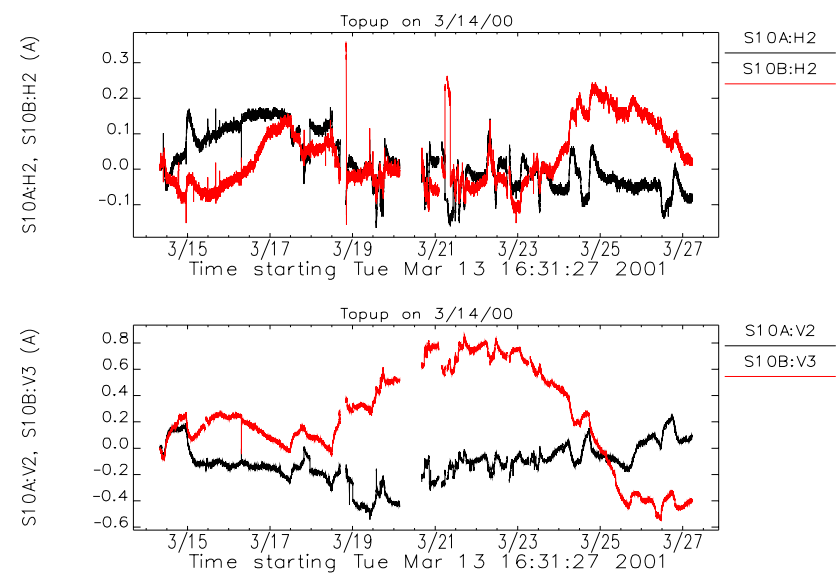

Figure 3: Slow variations of corrector effort in the same sector as Fig. 2 during the most recent top-up period of March 2001. The top graph shows the horizontal correctors, and the bottom graph shows the vertical correctors. One vertical corrector is correlated with the unexpected orbit drift shown in the second graph of Fig. 2.

consideration has been given to the XBPM data, considering that bad or missing data occur occasionally at particular beamlines. In addition, there may be changes in BPM readbacks caused by intentional steering of the beamline that should not be included as orbit drift. We would also like to exclude short-term glitching from the analysis. It is impractical to examine all the data manually and edit out the parts that should be excluded. Instead we keep all the data, but replace the average value with a median value. The median is not necessarily equal to the average of the good data, but it is insensitive to the distribution of bad data. These comments apply to the decile range value as a measure of spread instead of using, for example, the total spread or the rms. These statistical quantities are useful in characterizing the orbit drift and in comparing the same type of data taken under different conditions. 
The decile ranges of each XBPM or corrector quantity were calculated for all top-up periods, then the median of these ranges was found - reducing all the data to one number per device type per plane per run. The statistical results of a top-up run are compared against the results for the preceding 6-day refill mode period with the same analysis. Table 3 shows the median of the decile ranges for the XBPMs for the three top-up runs. Figure 4 shows the distribution of decile ranges for the top-up run of March 2001.

Table 1: Medians of Decile Range of XBPMs, and Vertical and Horizontal Correction

\begin{tabular}{|c|c|c|c|}
\hline Run & $\begin{array}{c}\text { XBPMs } \\
(\mu \mathrm{m})\end{array}$ & $\begin{array}{c}\text { V corr. } \\
(\mu \mathrm{rad})\end{array}$ & $\begin{array}{c}\text { H corr. } \\
(\mu \mathrm{rad})\end{array}$ \\
\hline 3/01 Re-fill & 14.0 & 1.8 & 0.9 \\
\hline 3/01 Top-up & 39.0 & 3.4 & 2.2 \\
\hline \hline 12/00 Re-fill & 15.4 & 2.6 & 1.6 \\
\hline 12/00 Top-up & 13.1 & 2.2 & 1.3 \\
\hline \hline 6/00 Re-fill & 13.8 & 3.9 & 2.0 \\
\hline 6/00 Top-up & 14.9 & 2,5 & 1.4 \\
\hline
\end{tabular}

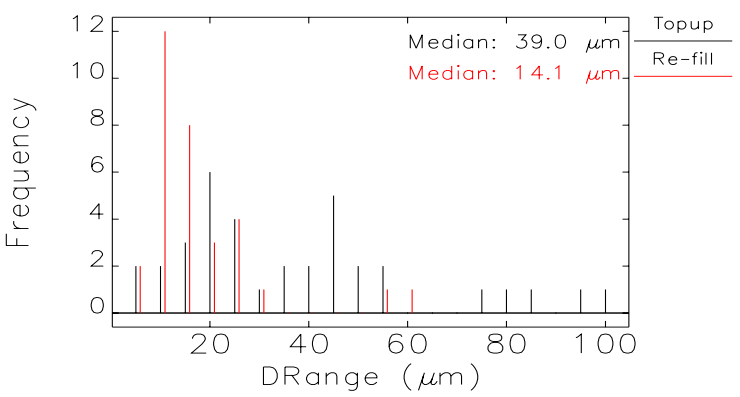

Figure 4: Histogram of decile ranges of XBPM readbacks for the most recent top-up period of March 2001 and the 6-day refill operation preceding it. The distribution is unexpectedly worse for top-up.

In the previous two top-up runs, we found improvements in the orbit drift. In the most recent one we find that, unexpectedly, the orbit drift is larger. For example, Fig. 2 shows that for a particular beamline, a smooth $100-\mu \mathrm{m}$ variation of XBPMs readbacks occured during the top-up period, which includes a 10 -h hiatus. Compare this variation to that in Fig. 5, which shows the same beamline for a previous top-up run (December 2000).

The same relatively large drift behavior applies to the vertical corrector analysis (see bottom graph of Fig. 3 . Somewhat smaller drifts occur in other beamlines, but they still compare badly to their respective refill periods. Examining rf BPM data in detail, we find that the beam intensity was indeed constant during the 13-day period (excluding short injector downtimes). Some rf BPMs in the sector showed little motion, while others showed motion consistent with the actions of the corrector. There are some rf BPMs for which the readbacks are known to be influenced by weak microwave modes inside the vacuum chamber. These BPMs are supposed to be ignored by orbit feed-

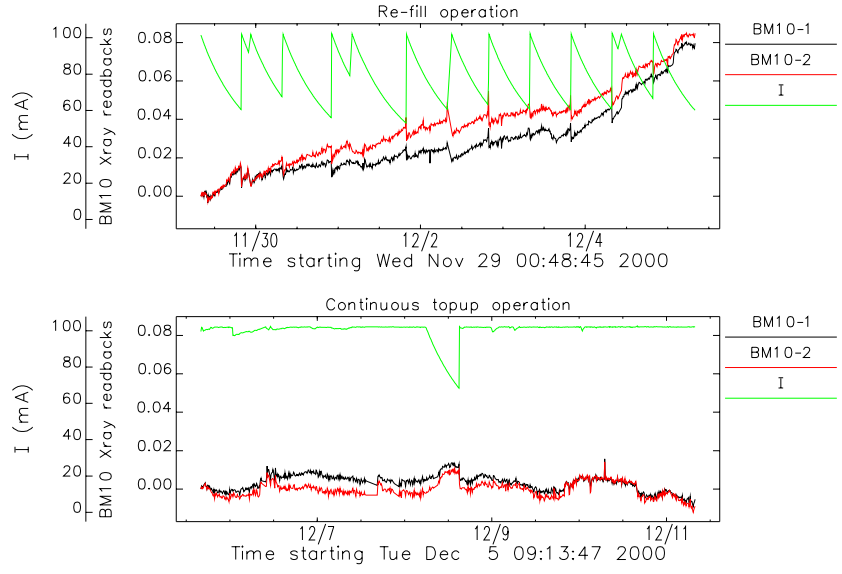

Figure 5: Variations of XBPMs (sector 10) during a past re-fill/top-up period of December 2000. The orbit drift is improved during top-up operations.

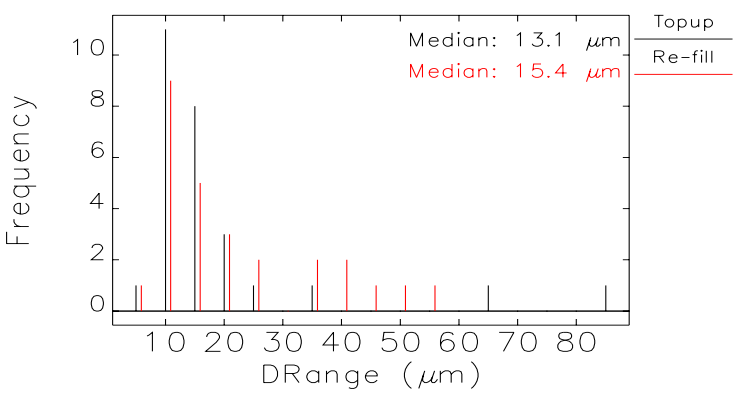

Figure 6: Histogram of decile ranges of XBPM readbacks for the past top-up period of December 2000 and the 6-day of re-fill operation preceding it. The distribution is better for the top-up running.

back through a special despiking filter that excludes any readbacks spiking above its neighbors by more than some preset value, e.g., $20 \mu \mathrm{m}$, determined from experience. In this case the bad rf BPMs outnumbered the good ones, and somehow "took over" orbit correction, moving the beam away at the beamline source point.

The effect of the microwave modes is subtly bunchpattern-dependent and apparently not always reproducible for the same approximate bunch pattern. The injection algorithm ensured that the pattern was preserved to within $0.25 \mathrm{~mA}(6 \%)$ for each bunch.

The problem can be fixed by removing some of the vertical BPMs from the orbit configuration and using the XBPMs in the orbit correction configuration, which has been done in the past successfully in a limited fashion.

The excessive orbit drift reported for the most recent topup run is not a problem specific to top-up mode, though we still do not know why it has not been observed in previous top-up runs.

Running top-up has not yielded the expected benefits easily but we believe that with careful investigations and additional top-up experience we will solve the problems. 